\title{
Prospective crowdsensing versus retrospective ratings of tinnitus variability and tinnitus-stress associations based on the TrackYourTinnitus mobile platform
}

\author{
Rüdiger Pryss ${ }^{1}\left(\mathbb{D} \cdot\right.$ Thomas Probst $^{2}\left(\mathbb{D} \cdot\right.$ Winfried Schlee $^{3} \cdot$ Johannes Schobel $^{1} \cdot$ Berthold Langguth $^{3}$. \\ Patrick Neff ${ }^{4} \cdot$ Myra Spiliopoulou $^{5} \cdot$ Manfred Reichert $^{1}$
}

Received: 28 September 2017 / Accepted: 24 February 2018 / Published online: 12 March 2018

(c) Springer International Publishing AG, part of Springer Nature 2018

\begin{abstract}
Many symptoms of neuropsychiatric disorders, such as tinnitus, are subjective and vary over time. Usually, in interviews or self-report questionnaires, patients are asked to retrospectively report symptoms as well as their severity, duration and influencing factors. However, only little is known to what degree such retrospective reports reflect the actual experiences made in daily life. Mobile technologies can remedy this deficiency. In particular, mobile self-help services allow patients to prospectively record symptoms and their severity at the time (or shortly after) they occur in daily life. In this study, we present results we obtained with the mobile crowdsensing platform TrackYourTinnitus. In particular, we show that there is a discrepancy between prospective and retrospective assessments. To be more precise, we show that the prospective variation of tinnitus loudness does not differ between the users who retrospectively rate tinnitus loudness as "varying" and the ones who retrospectively rate it as "non-varying." As another result, the subjectively reported stress-level was positively correlated with tinnitus (loudness and distress) in the prospective assessments, even for users who retrospectively rated that stress reduces their tinnitus or has no effect on it. The results indicate that mobile technologies, like the TrackYourTinnitus crowdsensing platform, go beyond the role of an assistive service for patients by contributing to more detailed information about symptom variability over time and, hence, to more elaborated diagnostics and treatments.
\end{abstract}

Keywords Prospective assessment of neuropsychiatric symptoms - Retrospective assessment of neuropsychiatric symptoms . Self-help mobile applications $\cdot$ Tinnitus $\cdot$ Mobile data collection $\cdot$ Mobile crowdsensing

\section{Introduction}

Rüdiger Pryss

ruediger.pryss@uni-ulm.de

Thomas Probst

Thomas.Probst@donau-uni.ac.at

Winfried Schlee

winfried.schlee@gmail.com

Johannes Schobel

johannes.schobel@uni-ulm.de

Berthold Langguth

berthold.langguth@medbo.de

Patrick Neff

patrick.neff@uzh.ch

Myra Spiliopoulou

myra@iti.cs.uni-magdeburg.de

Manfred Reichert

manfred.reichert@uni-ulm.de
The assessment of neuropsychiatric symptoms is essential in psychology, medicine and neuroscience. For many neuropsy-

1 Ulm University, James-Franck-Ring, 89081 Ulm, Germany

2 Department for Psychotherapy and Biopsychosocial Health, Danube University Krems, Dr.-Karl-Dorrek-Straße 30, 3500 Krems, Austria

3 Clinic and Policlinic for Psychiatry and Psychotherapy, University of Regensburg, Universitätsstraße 84, 93053 Regensburg, Germany

4 Neuroplasticity and Learning in the Healthy Aging Brain, University of Zurich, Andreasstrasse 15, 8050 Zurich, Switzerland

5 Department of Technical and Business Information Systems, Otto-von-Guericke-University Magdeburg, Universitätsplatz 2, 39106 Magdeburg, Germany 
chiatric disorders, the severity and duration of symptoms constitute essential criteria for diagnosis. For example, a major depressive episode can only be diagnosed if the patients have suffered from depressive symptoms for at least 2 weeks. Moreover, factors making the symptoms more or less strong (i.e., correlates of the symptoms) need to be identified for case conceptualization and treatment planning. Hence, psychologists, physicians and researchers need to reliably assess not only symptoms and their severity, but also their fluctuations over time. However, most indicators of the symptoms as well as their severity, duration and correlates are subjective. In current practice, usually, their assessment is based on retrospective reports of the patients. In turn, this raises the question to what degree patients are able to remember the severity, duration and correlates of the symptoms they have actually experienced.

Mobile technologies can effectively contribute to shed light on this question. In particular, they allow complementing the retrospective reports of the patients with prospective assessments of symptom variation over time. In this article, we describe how the mobile crowdsensing platform TrackYourTinnitus [31,32,36-38,42], which we developed during the last years, contributes to prospectively monitor symptom variability over time for individuals with tinnitus. Tinnitus can be described as the phantom perception of sound. Depending on its definition and duration as well as on the patient age and birth cohort, between 5.1 and $42.7 \%$ of the population worldwide experience tinnitus at least once during their lifetime [26]. On the one hand, tinnitus varies among patients (i.e., inter-individual variability); on the other hand, it may vary for a particular patient (i.e., intra-individual variability) as well. Moreover, the diagnosis and treatment of tinnitus and potential comorbidities require assessments of several symptoms like loudness and variation of the perceived sound(s), stress-level, depressive and anxiety symptoms as well as concentration. In this work, we aim to compare findings from prospective and retrospective assessments of tinnitus symptoms (loudness and distress) as well as the potential influencing factor/correlate "stresslevel," which is often reported in the context of tinnitus [24].

The limited (ecological) validity of retrospective selfreports has been shown in several studies on other neuropsychiatric disorders. For example, [1] assessed physical activities of patients with eating disorders by retrospective self-reports as well as prospective assessments with an accelerometer. Patients reported significantly less physical activity retrospectively compared to the prospective measurements with the accelerometer. In turn, [23] investigated retrospectively as well as prospectively assessed anxiety and related cognition in patients with agoraphobia. While anxiety did not differ between retrospective and prospective assessments, cognition did.
Such results highlight the importance of ecological momentary assessment (EMA; also known as ambulatory assessment \& experience sampling) to support clinicians in assessing neuropsychiatric symptoms as well as their correlates accurately in real time. In EMA, the variable in question (e.g., symptoms) is assessed repeatedly in daily life [46]. Instead of retrospectively asking the individuals, through an interview or questionnaire, how strongly they experienced a symptom in a given past time interval, the individuals are asked how they currently experience it as well as its severity and potential correlates. In turn, this is accomplished at several time points within the given time interval.

In the aforementioned studies, prospective and retrospective assessments were juxtaposed manually. To effectively exploit the prospective assessments in a clinical setting, however, an integrated solution is needed, i.e., the EMA of a patient should be transferred automatically to a database and be made available to the responsible clinician(s) [34,43], given the consent of the patient. Note that it has been already reported for a long time that electronic systems are appreciated by study participants [17], increase data accuracy [29], lead to more complete datasets [22] and reduce costs [30] compared to traditional paper-based methods. However, the exploitation of the prospective assessments next to the retrospective reports has not been investigated in the area of tinnitus yet.

This paper presents the TrackYourTinnitus (TYT) mobile crowdsensing platform $[37,38]$ for the juxtaposition of retrospective and prospective assessments, with a focus on tinnitus loudness, tinnitus distress and psychological stress. We elaborate how the prospective data are collected and how they should be maintained for further usage. The paper provides a significant extension of the work we presented in [35]. In particular, [35] did not include the analysis of tinnitus distress and psychological stress. As the latter is associated with several disorders (in general [3]; for tinnitus [24]), this analysis constitutes another fundamental comparison between real-time assessments and retrospective reports. We provide detailed backgrounds on the gathered and evaluated data as well as the results for psychological stress. This additional analysis reconfirms that mobile crowdsensing services will become increasingly important for collecting large and ecologically valid longitudinal datasets in the context of clinical research.

The remainder of the paper is organized as follows. Related work is discussed in Sect. 2. In Sect. 3, we describe the TYT mobile crowdsensing platform and explain the workflow we implemented for collecting and maintaining prospective assessments. Section 4 presents the data as well as the statistics used for juxtaposing the prospective with the retrospective assessments. In Sect. 5, we present the results of the statistical analyses, which suggest that integrating prospective assessments into the diagnostic-therapeutic 
process is crucial for optimizing diagnostics and, hence, treatments. The paper concludes with a summary and outlook in Sect. 6.

\section{Related work}

Mobile crowdsensing is an emerging research topic in various application domains $[19,20,39,45]$. In the medical domain, however, this research direction has been neglected so far. The fact that the medical domain is less considered by contemporary crowdsensing approaches might be explainable by legal and data privacy issues [2]. Nevertheless, mobile crowdsensing offers promising perspectives for the medical domain [8], as it exhibits unique features for gathering valuable patient data in the large scale [5]. In particular, mobile crowdsensing allows for the effective, context-aware gathering [21] of daily life patient data [33], which, in turn, will shift clinical research to a new level.

Besides TrackYourTinnitus (TYT), other studies have applied EMA approaches to track tinnitus in daily life $[9,11,27,48]$. Yet, their focus was not to compare retrospective ratings and prospectively crowdsensed data. [48] and [11] were pilot studies that showed for example that tinnitus tracking is feasible without negative consequences for the participants and that tinnitus varies within and between participants. [9] investigated fluctuations of tinnitus as well as associations between tinnitus and stress. [27] tracked tinnitus and other symptoms (e.g., dizziness) in patients with Meniere's syndrome. Contrary to these approaches, TYT is an open-source application, available for download in the iOS app store or Google play store, so that there is a larger and more representative sample compared to the participants of the cited studies.

Beyond tinnitus, EMA approaches capturing many other aspects such as pain [14] or feelings [15] in daily life were scientifically evaluated. In addition, EMA approaches were studied in the areas of mood disorders and mood dysregulation [40,47] as well as in the context of substance use [44] and eating disorders [7]. In psychotherapy research, EMA has been used to predict patient progress [13]. Although most neuropsychiatric symptoms are subjective experiences and, thus, most EMA approaches use self-reports to capture these symptoms, some neuropsychiatric symptoms are behavioral (e.g., avoidance in anxiety disorders) or physiological (e.g., increase of heart rate in anxiety disorders). Note that mobile systems offer opportunities to measure behavioral or physiological data in daily life [6].

Altogether, EMA approaches provide unprecedented opportunities to study neuropsychiatric symptoms under ecologically valid conditions [28], even though the utilization of its possibilities is still in its infancy, especially in the medical domain.

\section{The TrackYourTinnitus platform}

TrackYourTinnitus (TYT) is a mobile crowdsensing platform that comprises a Web site for user registration, two mobile applications (for iOS and Android) and a relational database (MySQL) as the central repository storing the collected data [37]. In particular, the anonymized or pseudonymized TYT data from the repository are made available to clinicians as well as researchers. The Web site further provides two fundamental features: First, users can visualize recorded tinnitus data; second, they can report on their current tinnitus treatment. In general, TYT was developed to track the individual tinnitus perception of users. In this context, the procedure depicted in Fig. 1 is applied to the TYT users. ${ }^{1}$

Following this procedure, TYT pursues three goals. First, data shall be collected on a daily basis (cf. Fig. 1, (3)). However, a crowd user shall not foresee the times he or she is asked to sense data (cf. Fig. 1, (2)). This is ensured by asking the crowd users in various daily life situations. Second, the collected data shall enable new kinds of data analytics like juxtaposing real-time assessments and retrospective reports (cf. Fig. 1, (1)). Third, gathered data shall be used to provide feedback to the mobile crowd users.

To enable the use of TYT as well as to provide data being appropriate for the data analysis applied in the context of this paper, the following procedure has to be accomplished by the users (cf. Fig. 1).

First, users have to create a TYT account, by using either the TYT Web site or the TYT mobile applications.

Second, users have to fill in three registration questionnaires (cf. Fig. 5). First of all, they have to fill in the "Mini-TQ-12" questionnaire (cf. Fig. 5, Mini-TQ-12 [12]), which measures tinnitus-related psychological problems. Second, they have to fill in the "Tinnitus Sample Case History Questionnaire" (TSCHQ) (cf. Fig. 5, TSCHQ [18]), in which details about the current tinnitus status, relevant co-morbidities and the tinnitus history are assessed. Note that TSCHQ comprises two questions being crucial for the results of this paper. To be more precise, the 11th item of TSCHQ asks the user retrospectively whether or not the tinnitus loudness varies from day to day. The 26th item of TSCHQ, in turn, requests from users to rate retrospectively whether stress is associated with their tinnitus. Third, users have to fill in the "Worst Symptom" questionnaire (cf. Fig. 5, Worst Symptom questionnaire), which asks the users about the worst symptom currently caused by their tinnitus. While the first two questionnaires constitute already used instruments, the third one was newly developed in the context of the presented research. Altogether, the completion of the three questionnaires with

\footnotetext{
$\overline{1}$ More detailed information about the procedure can be found at https:// www.trackyourtinnitus.org/process.pdf
} 


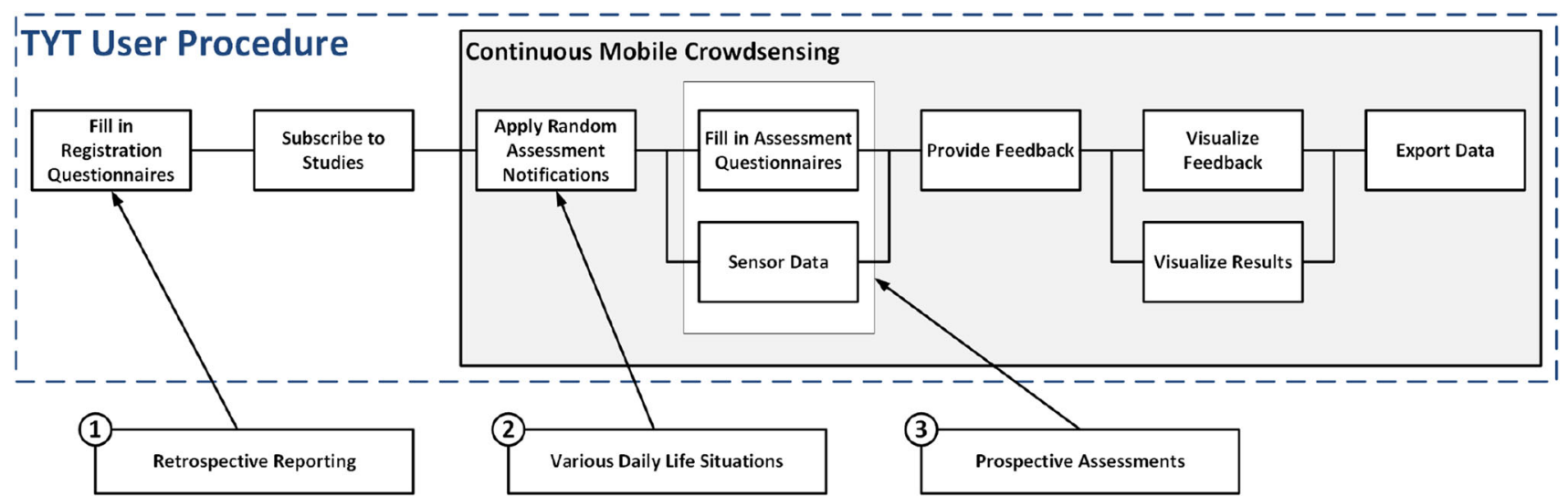

Fig. 1 Mobile crowdsensing collection procedure

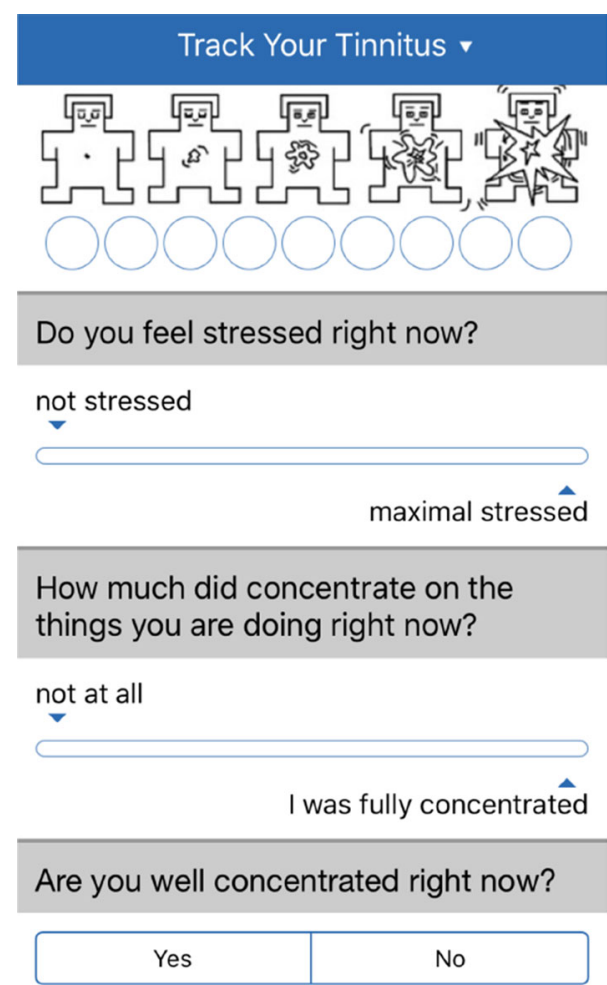

their 58 questions in total is a fundamental prerequisite for users who want to access the TYT Web site features as well as the TYT mobile applications (Fig. 2).

Third, after registering and completing the required questionnaires, users may exploit the mobile applications to track their tinnitus and potential correlates during daily life. For this purpose, a user needs to $\log$ in to the Android or iOS mobile application. Then, he/she is asked to fill in the assessment questionnaire developed for TYT (cf. Fig. 5, assessment questionnaire). This repeatedly administered assessment questionnaire comprises 8 items (cf. Table 1) including questions on the current tinnitus loudness, tinnitus distress and subjective stress-level. Figure 3 gives an impression of how the questionnaire looks like in iOS.

Fourth, the assessment questionnaire is provided in two ways. Either the mobile application automatically displays the questionnaire to the user or the user himself makes the conscious decision to fill out the questionnaire (cf. Fig. 5, conscious decision). The first procedure is the preferred one and is realized as follows: The assessment questionnaire is randomly presented to the user up to 12 times per day. Figure 3 gives an impression of the notification settings in iOS.

Fig. 2 Impression of assessment questionnaire in iOS

Table 1 TrackYourTinnitus assessment questions

\begin{tabular}{llll}
\hline & Question & Scale & M \\
\hline (1) & Did you perceive the tinnitus right now? & BS & Perception \\
(2) & How loud is the tinnitus right now? & VAS & Loudness \\
(3) & How stressful is the tinnitus right now? & VAS & Strain \\
(4) & How is your mood right now? & VAS & Mood \\
(5) & How is your arousal right now? & VAS & Arousal \\
(6) & Do you feel stressed right now? & VAS & Stress \\
(7) & How much did you concentrate on the & & Con. \\
& things you are doing right now? & BS & Irritability \\
\hline
\end{tabular}

$B S$ binary scale, VAS visual analogue scale, $M$ measurement of, Con. concentration 


Settings
Activate Notifications
Schedule
Standard
You get notified in a random-like manner.
First set the maximum number of
notifications you want to receive.
Notifications per day 12 -
Activation Schedule
Please select the time span per day below
in which you want to receive notifications.
Monday $22: 00$
Tuesday $08: 00$ - $08: 00$ -

Fig. 3 Impression of notification settings in iOS

For the application of the assessment questionnaire, notification features for both Android and iOS as well as a notification algorithm were realized [37]. We only present the algorithm running on iOS (cf. Algorithm 1) and the calculated notifications for a single day. In practice, notifications are calculated in advance. The algorithm, in turn, works as follows:

1. It partitions the time window a user has specified with respect to a particular day into $n$ time intervals of equal length. $n$ corresponds to the number of notifications the user has chosen.

2. The algorithm then calculates exactly one notification for each interval. Thereby, it ensures that for each notification the points in time are randomly calculated.

3. Finally, it is ensured that there is an interval of at least 15 min between two notifications.

On the one hand, the procedure ensures that users cannot foresee the time when being asked; on the other hand, it ensures that they are sensed in various daily situations. Note that this randomized approach was realized to improve the ecological validity of the applied method. The approach to randomly apply the assessment questionnaire is illustrated in

\footnotetext{
2 arc4random_uniform(upper_bound): iOS internal function to return a uniformly distributed random number less than upper_bound.
}
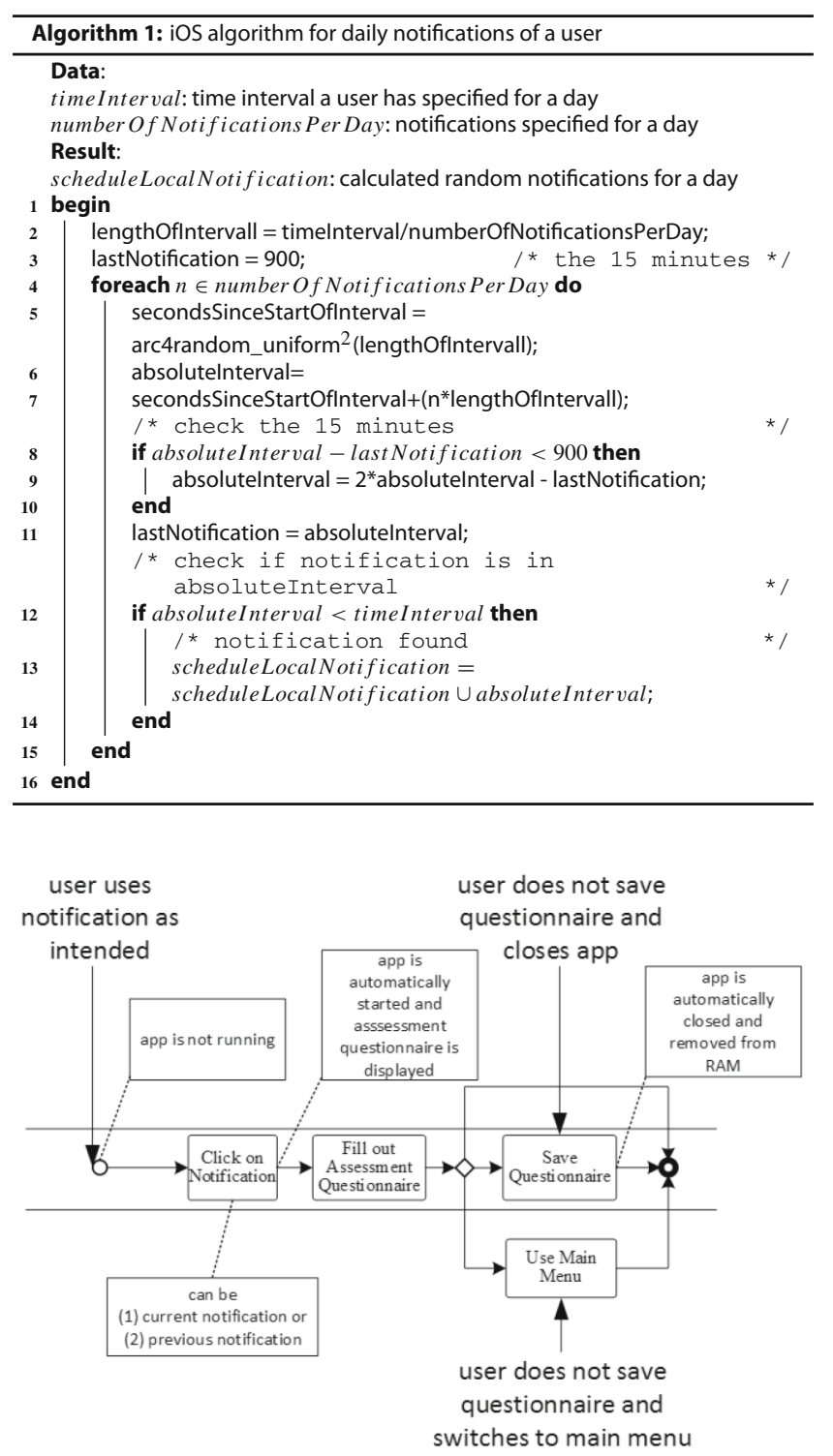

Fig. 4 Possible user action after a notification

Fig. 4. It works on both mobile operating systems in exactly the same ways.

After a notification appears, the user may click on it. In the latter case, the TYT mobile app is started (if not already running) and the assessment questionnaire is directly displayed to the user. Then, he or she can fill out the questionnaire and finally save the entered data. After saving the questionnaire data, the mobile app is terminated $3 \mathrm{~s}$ later. Within these $3 \mathrm{~s}$ the result is transferred to the TYT backend (if the mobile app is online; otherwise, the result is locally stored until the device gets an online connection).

The procedure to automatically terminate the app shall speed up the process to fill out questionnaires after being notified. Note that the user feedback we have received so far supports this technical procedure. If the user does not save 


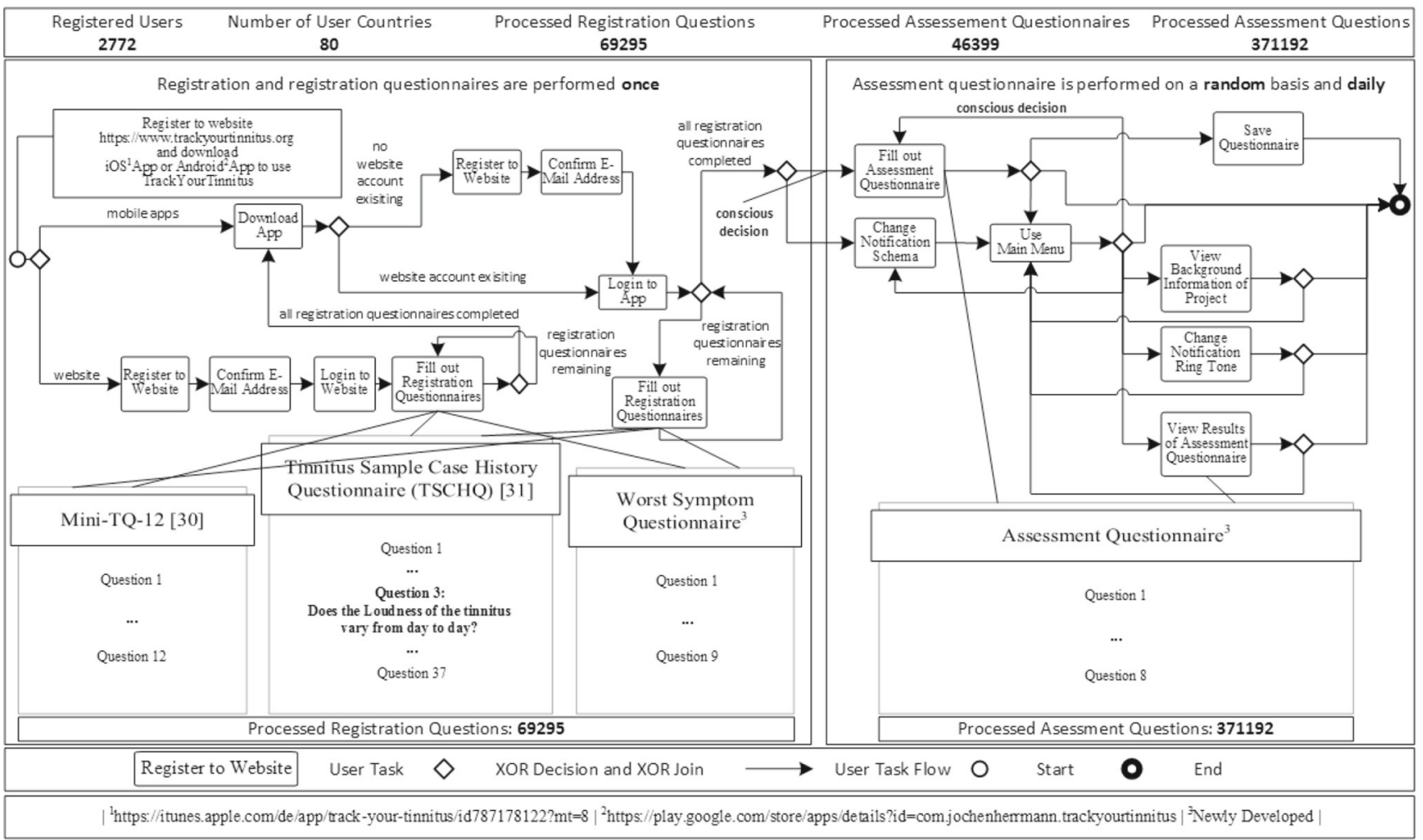

Fig. 5 TrackYourTinnitus crowdsensing platform

the questionnaire data or switches to the main menu before saving it, the questionnaire will be not saved and transferred to the TYT backend.

Finally, another aspect related to notifications is important. As can be seen from Fig. 4, the user might click on a current notification or previous ones. The latter situation might occur if the user did not click on a previous notification. Each time a notification is not clicked, the TYT mobile app memorizes and stores it in the local message store. If a user clicks on the local message store, he or she may see these previous notifications. Note that the allowed amount of previous notifications messages differs between the two mobile operating systems. As we store separate timestamps for the notification as well as the saving time of each processed assessment questionnaire, users can also fill out questionnaires related to previous notifications.

Fifth, while filling in the assessment questionnaire, the smart mobile device of a user records the environmental sound level if this option has been activated.

Sixth, results gathered with the assessment questionnaire and the sound-recording component are transferred to the TYT database. The latter, in turn, provides comprehensive features that enable researchers to evaluate the gathered user data. Note that we exactly used these features to obtain the results presented in Sect. 5.
The TYT crowdsensing platform provides multilingual support. Currently, English, German and Dutch are supported. The current number (September 2017) of processed questionnaires and registered users is shown in Fig. 5. As can be seen, the TYT mobile crowdsensing platform is frequently used. So far, users from 80 countries have entered data to the platform. It is noteworthy that users from seven countries provide the major part of all data gathered. To be more precise, most of the collected data is provided by users from Germany, followed by the USA, Netherlands, the UK, Switzerland, Canada and France.

In the following, technical insights into the TYT platform, which are relevant in the context of this work, will be sketched. Concerning domain-specific requirements (from the medical and psychological domains), the TYT platform stores user data in an anonymized or pseudonymized manner. Only if users want to use the password reset function, they have to provide personal data (i.e., the e-mail address), which are saved separately from the app data. However, users are informed about the fact that the e-mail address must be stored for resetting a password.

To ensure privacy, TYT presents a consent form to users. Only if users confirm the consent form, the respective function is used by TYT. For example, if access to mobile device features is required (e.g., to record the sound level by the mobile device microphone), users must explicitly confirm 
this through a consent form. Another feature provided by TYT is the participation in studies. Users may enter or leave studies. Each study, in turn, may rely on questionnaires, consent forms and notifications settings. Results of processed questionnaires are always locally stored as JSON-file if there is no Internet connection. If the mobile device has an Internet connection, results are transferred as JSON-files through a REST-API to the TYT backend. The latter stores them in a relational database.

For researchers, a backend function is provided that enables them to export the data from the relational database to CSV files. The export feature, in turn, particularly considers that exported data are processed by different statistical software systems. For example, all the data of a user can be stored in one row with multiple columns or data can be stored in a way so that one assessment point represents one row. Recently, TYT was enhanced with data from wearables (e.g., measuring physiological data like the heart rate). We integrated the iOS smart watch and fitness trackers offered by GARMIN and MIO to the TYT platform. Respective trackers can be used to track the heart rate while filling out the assessment questionnaire. First results related to these heart rate measurements are promising. In addition, we provide a feature to determine the GPS position while filling out the assessment questionnaire.

In the meantime, the basic design principles, techniques and components of TYT were adopted in other contexts as well. For example, in the myKind project [39], risk factors during pregnancy are tracked and evaluated based on the TYT technology. Another project using the TYT technology is TrackYourHearing ${ }^{2}$, which aims to gain new insights into the moment-to-moment variability of the hearing loss of users.

Altogether, TYT constitutes a powerful, generic framework for measuring the moment-to-moment variability of user aspects in everyday life.

\section{Data and statistics}

The analysis presented in this section is based on an export of the TYT database made in August $2017 .{ }^{3}$ In the current study, we apply different statistical approaches to investigate the following two research questions:

1. Does the variation of prospectively assessed tinnitus loudness differ between TYT users who rated their tinnitus loudness retrospectively as varying and TYT users who rated their tinnitus loudness retrospectively as nonvarying?

\footnotetext{
2 https://www.trackyourhearing.org

${ }^{3} N=1491$ users completed at least one assessment questionnaire and provided meaningful datasets for the analysis.
}

2. How is the prospectively assessed subjective stress-level associated with (a) prospectively assessed tinnitus loudness and (b) prospectively assessed tinnitus distress in the following three user groups: users retrospectively rating that stress worsens their tinnitus, users retrospectively rating that stress reduces their tinnitus and users retrospectively rating that stress has no effect on their tinnitus?

Regarding research question 1, two variables were investigated. The first one assessed the variability of tinnitus loudness retrospectively. The following TSCHQ item, which was asked once during the TYT registration process, was used to measure retrospectively whether or not the tinnitus loudness varies: "Does the LOUDNESS of the tinnitus vary from day to day?" with the options to respond with either "yes" or "no." The second variable measured tinnitus loudness prospectively; at several points in time during their daily life, the users rated the following question of the assessment questionnaire: "How LOUD is the tinnitus right now?," with a slider ranging from 0 (i.e., not audible) to 1 (i.e., maximal loudness). To enable a comparison of the retrospective assessments on day-to-day variation with the variation of the prospective assessments, we had to focus our analysis on the day-to-day variation (i.e., excluding within-day variation) of the prospective assessments. Therefore, for each patient with more than one prospective tinnitus loudness assessment per day, we calculated the mean of the within-day prospective assessments in order to have one prospective loudness assessment per day. Then, the variability of these prospective loudness ratings was calculated for each user as the standard deviation (SD) of all their prospective loudness ratings (on a day-to-day level). To obtain a meaningful day-to-day variability of the prospective tinnitus loudness ratings, only those users who provided a prospective tinnitus loudness assessment at least at 10 days were investigated. We used a t test for independent samples (two-tailed, with $p<0.05$ indicating a statistically significant result) to evaluate whether the SD of the prospective tinnitus loudness assessments differs between users retrospectively describing their tinnitus loudness as non-varying and users retrospectively describing their tinnitus loudness as varying.

After applying these criteria, plus the exclusion of assessments with an inter-assessment interval of 15 mins or less [37], $N=305$ users remained for the statistical analysis. Of these $N=305$ users, $n=54$ (18\%) reported retrospectively that the tinnitus loudness does not vary from day to day and $n=251(82 \%)$ reported retrospectively that the tinnitus loudness varies from day to day. While the users retrospectively rating the tinnitus loudness as stable provided prospective assessments of tinnitus loudness for $M=40.48$ ( $S E=5.79$ ) days (in an average interval of $M=4.25$ months), the users retrospectively rating the tinnitus as vary- 
ing provided data for $M=44.26(S E=3.62)$ days (in an average interval of $M=4.34$ months).

We also ran the statistics for users with prospective loudness assessments at least at 25 days in order to investigate whether the results can be replicated. After applying these stricter inclusion criteria, $N=158$ users were remaining for the statistical analysis. Of these users, $n=29$ (18\%) reported retrospectively that the tinnitus loudness does not vary from day to day, whereas $n=129(82 \%)$ reported retrospectively that the tinnitus loudness varies from day to day. Here, the users retrospectively rating the tinnitus loudness as stable provided prospective assessments of tinnitus loudness for $M=61.38(S E=9.17)$ days (average time interval the assessments were provided in: $M=4.33$ months), whereas the users retrospectively rating the tinnitus as varying provided data for $M=71.80(S E=6.13)$ days (average time interval the assessments were provided in: $M=4.98$ months). In order to investigate whether the TYT users retrospectively rating the tinnitus loudness as non-varying differ in baseline variables from users retrospectively rating the tinnitus loudness as varying, independent $t$ tests and chi-squared tests were performed (again, two-tailed with $p<0.05$ as statistical significance level).

Regarding research question 2, four variables were evaluated. The first one assessed the impact of stress on tinnitus retrospectively. The following TSCHQ item, again asked once during the TYT registration procedure, was used to measure retrospectively how the stress-level affects tinnitus: "Does stress influence your tinnitus?" with the options to respond "has no effect," "reduces my tinnitus" or "worsens my tinnitus." This first variable was available only for a subgroup of the TYT users $(N=675)$. Of these $N=675$ users, $n=419$ (62\%) rated "worsens my tinnitus," $n=20$ (3\%) "reduces my tinnitus" and $n=236(35 \%)$ "has no effect." The second variable is prospectively measured tinnitus loudness. As described above, at several time points during their daily life, TYT users rated the following question: "How LOUD is the tinnitus right now?" with a slider ranging from 0 (i.e., not audible) to 1 (i.e., maximal loudness). The third variable is prospectively measured tinnitus distress. Hereby, the following question was rated at several time points by TYT users during their daily life "How STRESSFUL is the tinnitus right now?" with a slider ranging from 0 (i.e., not distressed) to 1 (i.e., maximal distress). The fourth variable is the prospectively assessed stress-level. The question "Do you feel stressed right now?" was rated with a slider ranging from 0 (i.e., no stress-level) to 1 (i.e., maximal stress-level) at several time points by TYT users during their daily life. Thereby, the $2 \mathrm{nd}$, 3rd and 6th variable was contained in the assessment questionnaire.

To evaluate how the prospectively assessed stress-level is associated with prospectively assessed tinnitus (loudness and distress) for users who retrospectively rate that stress worsens their tinnitus, for users who retrospectively rate that stress reduces their tinnitus and for users who retrospectively rate that stress has no effect on their tinnitus, linear multilevel models were performed due to the nested structure of the data. The multilevel models had 2 levels: assessments as level- 1 and users as level-2. The multilevel models were performed with the full maximum likelihood estimation to handle missing data in the dependent variables (tinnitus loudness and tinnitus distress), and a random intercept term was included. The prospectively measured stress-level functioned as time-varying covariate in these multilevel models. Due to missing data in the covariate (no prospective stress-level assessment), $n=406$ users retrospectively reporting "stress worsens my tinnitus," $n=19$ users retrospectively reporting "stress reduces my tinnitus" and $n=230$ users retrospectively reporting "stress has no effect" could be analyzed with the multilevel models. Moreover, a chi-squared tests and ANOVAs were performed to compare the three groups (TYT users retrospectively rating that stress worsens their tinnitus, TYT users retrospectively rating that stress reduces their tinnitus and TYT users retrospectively rating that stress has no effect on their tinnitus) in baseline variables. Again, the statistical tests were performed two-tailed with $p<0.05$ as statistical significance level.

\section{Results}

This section presents the results we obtained with respect to the aforementioned two research questions.

Concerning research question 1, the comparisons between the users retrospectively rating the tinnitus as not varying and the users retrospectively rating the tinnitus as varying are given in Table 2. As can be seen, TYT users who retrospectively rated their tinnitus loudness as non-varying from day to day did not significantly differ in the average variation (SD) of the prospective tinnitus loudness assessments from the TYT users who retrospectively rated their tinnitus loudness as varying from day to day. This result emerged when TYT users providing prospective tinnitus loudness assessments at least at 10 days were analyzed as well as when TYT users providing prospective tinnitus loudness assessments at least at 25 days were analyzed.

Moreover, no significant differences in baseline variables (i.e., gender, age and tinnitus duration) were found between users with retrospectively varying and users with retrospectively non-varying tinnitus loudness. However, male users tended to be more often in the group retrospectively rating tinnitus loudness as non-varying than in the group retrospectively rating tinnitus loudness as varying $(p=0.053)$ in the sample consisting of TYT users with at least 10 prospective tinnitus loudness assessments; in the sample of TYT users with at least 25 prospective tinnitus loudness assess- 
Table 2 Results of research question 1

\begin{tabular}{|c|c|c|c|}
\hline & $\begin{array}{l}\text { Retrospective rating } \\
\text { of tinnitus loudness } \\
\text { variation = no }\end{array}$ & $\begin{array}{l}\text { Retrospective rating } \\
\text { of tinnitus loudness } \\
\text { variation = yes }\end{array}$ & Statistics \\
\hline \multicolumn{4}{|c|}{ TYT users with prospective tinnitus loudness ratings at least at 10 days } \\
\hline Male gender $n(\%)$ & $44(83.0)$ & $174(69.9)$ & $\chi^{2}(1)=3.757 ; p=0.053$ \\
\hline Age $M(\mathrm{SE})$ & $38.262(3.337)$ & $44.426(1.306)$ & $t(292)=-1.597 ; p=0.111$ \\
\hline $\begin{array}{l}\text { Tinnitus duration (subjective report in } \\
\text { years) M (SE) }\end{array}$ & $13.093(2.032)$ & $11.142(0.752)$ & $t(65.666)=0.901 ; p=0.371$ \\
\hline Mini-TQ-12 [12] score M (SE) & $12.462(0.749)$ & $13.544(0.356)$ & $t(298)=-1.275 ; p=0.203$ \\
\hline $\begin{array}{l}\text { Variation (SD) of the prospective } \\
\text { tinnitus loudness assessments M (SE) }\end{array}$ & $0.151(0.009)$ & $0.150(0.004)$ & $t(303)=0.106 ; p=0.916$ \\
\hline \multicolumn{4}{|c|}{ TYT users with prospective tinnitus loudness ratings at least at 25 days } \\
\hline Male gender $n(\%)$ & $24(82.8)$ & $93(72.7)$ & $\chi^{2}(1)=1.271 ; p=0.260$ \\
\hline Age $M(\mathrm{SE})$ & $34.385(5.603)$ & $45.657(1.794)$ & $t(31.534)=-1.916 ; p=0.064$ \\
\hline $\begin{array}{l}\text { Tinnitus duration (subjective report in } \\
\text { years) } M(S E)\end{array}$ & $16.364(2.974)$ & $11.353(1.047)$ & $t(33.991)=1.589 ; p=0.121$ \\
\hline Mini-TQ-12 [12] score M (SE) & $12.793(0.986)$ & $13.024(0.451)$ & $t(154)=-0.219 ; p=0.827$ \\
\hline $\begin{array}{l}\text { Variation (SD) of the prospective } \\
\text { tinnitus loudness assessments M (SE) }\end{array}$ & $0.150(0.013)$ & $0.136(0.006)$ & $t(156)=1.053 ; p=0.294$ \\
\hline
\end{tabular}

Results of the comparisons between TYT users retrospectively rating the tinnitus loudness as non-varying and TYT users retrospectively rating the tinnitus loudness as varying

TYT TrackYourTinnitus, $M$ mean, $S D$, standard deviation, Mini-TQ tinnitus questionnaire short form

Table 3 Results of research question 2

\begin{tabular}{|c|c|c|c|c|c|c|}
\hline Variable & Parameter & Estimate & SE & $d f$ & $T$ statistic & $p$ value \\
\hline \multicolumn{7}{|c|}{ Users retrospectively rating that stress worsens their tinnitus } \\
\hline \multirow[t]{2}{*}{ Tinnitus loudness } & $\begin{array}{l}\text { Intercept (tinnitus loudness when } \\
\text { statistically controlling for stress-level) }\end{array}$ & 0.397 & 0.011 & 444.752 & 35.989 & $<0.001$ \\
\hline & Influence of the stress-level & 0.343 & 0.010 & $12,372.290$ & 35.191 & $<0.001$ \\
\hline \multirow[t]{2}{*}{ Tinnitus distress } & $\begin{array}{l}\text { Intercept (tinnitus distress when } \\
\text { statistically controlling for stress-level) }\end{array}$ & 0.271 & 0.010 & 419.144 & 27.897 & $<0.001$ \\
\hline & Influence of the stress-level & 0.458 & 0.009 & $12,239.407$ & 52.375 & $<0.001$ \\
\hline \multicolumn{7}{|c|}{ Users retrospectively rating that stress reduces their tinnitus } \\
\hline \multirow[t]{2}{*}{ Tinnitus loudness } & $\begin{array}{l}\text { Intercept (tinnitus loudness when } \\
\text { statistically controlling for stress-level) }\end{array}$ & 0.376 & 0.056 & 24.649 & 6.716 & $<0.001$ \\
\hline & Influence of the stress-level & 0.342 & 0.061 & 179.736 & 5.597 & $<0.001$ \\
\hline \multirow[t]{2}{*}{ Tinnitus distress } & $\begin{array}{l}\text { Intercept (tinnitus distress when } \\
\text { statistically controlling for stress-level) }\end{array}$ & 0.360 & 0.054 & 24.267 & 6.721 & $<0.001$ \\
\hline & Influence of the stress-level & 0.319 & 0.061 & 181.532 & 5.196 & $<0.001$ \\
\hline \multicolumn{7}{|c|}{ Users retrospectively rating that stress has no effect on their tinnitus } \\
\hline \multirow[t]{2}{*}{ Tinnitus loudness } & $\begin{array}{l}\text { Intercept (tinnitus loudness when } \\
\text { statistically controlling for stress-level) }\end{array}$ & 0.377 & 0.016 & 252.331 & 24.269 & $<0.001$ \\
\hline & Influence of the stress-level & 0.388 & 0.016 & 6055.243 & 24.582 & $<0.001$ \\
\hline \multirow[t]{2}{*}{ Tinnitus distress } & $\begin{array}{l}\text { Intercept (tinnitus distress when } \\
\text { statistically controlling for stress-level) }\end{array}$ & 0.243 & 0.013 & 221.250 & 18.647 & $<0.001$ \\
\hline & Influence of the stress-level & 0.472 & 0.013 & 6032.276 & 35.194 & $<0.001$ \\
\hline
\end{tabular}

Results of the multilevel models on the associations between prospectively assessed stress-level and prospectively assessed tinnitus (loudness and distress) in TYT users retrospectively rating that stress worsens their tinnitus, in TYT users retrospectively rating that stress reduces their tinnitus and in TYT users retrospectively rating that stress has no effect on their tinnitus

$T Y T$ TrackYourTinnitus, $S D$ standard deviation, $d f$ degree of freedom 
Table 4 Results of research question 2: baseline variables

\begin{tabular}{|c|c|c|c|c|}
\hline & $\begin{array}{l}\text { TYT users retrospectively } \\
\text { rating that stress } \\
\text { worsens their tinnitus }\end{array}$ & $\begin{array}{l}\text { TYT users retrospectively } \\
\text { rating that stress } \\
\text { reduces their tinnitus }\end{array}$ & $\begin{array}{l}\text { TYT users retrospectively } \\
\text { rating that stress has no } \\
\text { effect on their tinnitus }\end{array}$ & Statistics \\
\hline Male gender $n(\%)$ & $288(68.7)$ & $16(80.0)$ & $166(70.6)$ & $\chi^{2}(2)=1.288 ; p=0.525$ \\
\hline Age $M(\mathrm{SE})$ & $41.509(0.813)$ & $26.103(4.590)$ & $42.576(1.337)$ & $F(2 ; 650)=-7.047 ; p=0.001$ \\
\hline $\begin{array}{l}\text { Tinnitus duration } \\
\text { (subjective report in } \\
\text { years) } \mathrm{M}(\mathrm{SE})\end{array}$ & $9.580(0.534)$ & $10.791(3.567)$ & $9.055(0.775)$ & $F(2 ; 643)=-0.296 ; p=0.744$ \\
\hline $\begin{array}{l}\text { Mini-TQ-12 [12] score } \\
\text { M (SE) }\end{array}$ & $14.057(0.290)$ & $13.500(1.192)$ & $11.644(0.386)$ & $F(2 ; 669)=12.590 ; p<0.001$ \\
\hline
\end{tabular}

Results of the comparisons between TYT users retrospectively rating that stress worsens their tinnitus, TYT users retrospectively rating that stress reduces their tinnitus and TYT users retrospectively rating that stress has no effect on their tinnitus

ments, the users retrospectively rating their tinnitus loudness as stable tended to be younger than the users retrospectively rating their tinnitus loudness as varying $(p=0.064)$. In summary, prospective assessments offer information not covered by retrospective ratings. This makes prospective assessments valuable for diagnostics and treatments.

Concerning research question 2, Table 3 summarizes the results of the multilevel models performed to evaluate the influence of the prospectively assessed stress-level on prospectively assessed tinnitus (loudness and distress) in users who retrospectively rate that stress worsens their tinnitus, in users who retrospectively rate that stress reduces their tinnitus and in users who retrospectively rate that stress has no effect on their tinnitus. It can be seen that the prospectively assessed stress-level was significantly positively associated with prospectively assessed tinnitus, both loudness and distress, in all multilevel models. Regardless of the TYT users' retrospective answer to the question "Does stress influence your tinnitus?" (worsens tinnitus, reduces tinnitus, no effect on tinnitus), a higher stress-level was significantly correlated with louder and more distressing tinnitus (all analyses: $p<0.001$ ).

The comparisons regarding baseline variables are presented in Table 4. Significant differences emerged in age- and tinnitus-related psychological problems as measured with the Mini-TQ score $(p \leq 0.001)$. The users retrospectively rating that stress reduces their tinnitus were younger, and the users retrospectively rating that stress has no effect on their tinnitus had the lowest tinnitus-related psychological problems. In particular, the finding that users retrospectively rating that stress reduces their tinnitus were younger should be investigated in more detail in further studies. As can be obtained from Table 4, the sample size of this group (third column) is much smaller than the samples of the other two groups and, hence, this result must be reevaluated with more TYT users in future. However, these preliminary results constitute promising results obtained through the use of mobile crowdsensing techniques.

\section{Summary and outlook}

The present study used data from a mobile crowdsensing platform, which we developed for tracking tinnitus in daily life for the juxtaposition of retrospective and prospective assessments.

First, retrospective and prospective measurements of variations of tinnitus loudness were compared with each other. As a result, we could show that the prospectively measured variation of tinnitus loudness does not significantly differ between individuals who retrospectively rated their tinnitus loudness as non-varying and individuals who retrospectively rated their tinnitus loudness as varying.

Second, the retrospective association between stress-level and tinnitus was contrasted with the prospective association between stress-level and tinnitus. The analysis of the prospective stress-level and tinnitus assessments showed that stress is associated with louder and more distressing tinnitus in TYT users who retrospectively rate that stress worsens their tinnitus as well as in TYT users who retrospectively rate that stress has no effect on their tinnitus and even in TYT users who retrospectively rate that stress reduces their tinnitus. However, it should be kept in mind that we investigated only cross-sectional associations between prospectively assessed tinnitus and prospectively assessed stress-level. This means that the stress-level at assessment $t$ was correlated with the tinnitus at $t$. It could be that different results might be visible when evaluating time-lagged effects the stress-level (assessment $t$ ) exerts on tinnitus (assessment $t+x$ ) or when the impact of changes of stress-levels (from assessment $t-2$ to assessment $t-1$ ) on subsequent changes of tinnitus (from assessment $t-1$ to assessment $t$ ) is analyzed. Corresponding research questions should be addressed in further studies, e.g., by using "latent difference score models" $[10,25]$.

In general, the results of the study at hand are in line with other studies showing that retrospective self-reports differ from prospective assessments. In particular, note that retrospective assessments might be biased (i.e., recall bias). 
Another explanation in the context of our study could be that the individuals who retrospectively rated their tinnitus as nonvarying and not worsened by stress have neither understood the ups and downs nor the correlates of their tinnitus yet. In psychotherapies with patients suffering from neuropsychiatric symptoms, a first technique often applied is to assign the homework to the patients to observe their symptoms in order to create an awareness that they fluctuate as well as to learn which factors influence the fluctuations. Possibly, individuals who retrospectively rate symptoms as varying and worsened by stress are more trained in self-observing strategies. In this context, it could be speculated that a longer tinnitus duration increases self-observation. Yet, our results do not support this as tinnitus duration was not significantly different between the investigated groups. Nevertheless, individuals who retrospectively assess tinnitus as non-varying and as not worsened by stress might gain a deeper understanding of the symptom when receiving feedback on the information that the symptom is prospectively varying and prospectively correlated with stress.

As another noteworthy aspect, in the presented study, we compare the real-time assessments (i.e., prospective reporting) with only one retrospective reporting that is gathered when TYT is executed for the first time by a user. If the real-time assessments make the user aware of how his or her tinnitus varies and how it is affected by the stress-level, the user's subjective evaluation of the retrospective reporting might change. This issue should be considered in future studies. In addition, we plan to investigate whether it is helpful for individuals with tinnitus to receive feedback on tinnitus fluctuations as well as on correlates (such as stress-level) as stored in the TYT platform.

A database of EMA assessments could be used to feed the patients' experiences of neuropsychiatric symptoms in daily life back to the responsible clinician(s) (in case the patient agrees with this procedure). This might be helpful to improve the treatment [16] or to support the clinicians in making diagnoses or case conceptualizations. The diagnostic procedure of neuropsychiatric symptoms is usually time- and cost-intensive. Time and costs, in turn, might be saved when patients use mobile systems in daily life to assess neuropsychiatric symptoms prospectively before the appointment for the diagnostic procedure takes place: clinicians, who receive feedback on these assessments, could adjust the diagnostic procedures to the data and would neither have to evaluate the patients' neuropsychiatric symptoms nor their correlates retrospectively. Finally, in future projects, the TYT crowdsensing platform will be extended with features that might reveal additional findings (e.g., through the application of gamification techniques [41] or forum features [4]). In summary, mobile crowdsensing systems capturing neuropsychiatric symptoms prospectively in daily life appear to offer several advantages that warrant further investigation and major breakthroughs in medical research.

\section{Compliance with ethical standards}

Conflict of Interest On behalf of all authors, the corresponding author states that there is no conflict of interest.

\section{References}

1. Bratland-Sanda, S., Sundgot-Borgen, J., Rø, Ø., Rosenvinge, J., Hoffart, A., Martinsen, E.: I'm not physically active-I only go for walks: physical activity in patients with longstanding eating disorders. Int. J. Eat. Disord. 43(1), 88-92 (2010)

2. Christin, D., Reinhardt, A., Kanhere, S., Hollick, M.: A survey on privacy in mobile participatory sensing applications. J. Syst. Softw. 84(11), 1928-1946 (2011)

3. Cohen, S., Janicki-Deverts, D., Miller, G.: Psychological stress and disease. JAMA 298(14), 1685-1687 (2007)

4. Dandage, S., et al.: Patient empowerment through summarization of discussion threads on treatments in a patient self-help forum. In: Maglaveras, N., Chouvarda, I., de Carvalho, P. (eds.) Precision Medicine Powered by pHealth and Connected Health. IFMBE Proceedings, vol. 66, pp. 229-233. Springer, Singapore (2018)

5. Demirbas, M., Bayir, M., Akcora, C., Yilmaz, Y., Ferhatosmanoglu, H.: Crowd-sourced sensing and collaboration using twitter. In: International Symposium on a World of Wireless Mobile and Multimedia Networks, pp. 1-9. IEEE (2010)

6. Ebner-Priemer, U., Kubiak, T.: Psychological and psychophysiological ambulatory monitoring. Eur. J. Psychol. Assess. 23(4), 214-226 (2007)

7. Engel, S., Crosby, R., Thomas, G., Bond, D., Lavender, J., Mason, T., Steffen, K., Green, D., Wonderlich, S.: Ecological momentary assessment in eating disorder and obesity research: a review of the recent literature. Curr. Psychiatry Rep. 18(4), 1-9 (2016)

8. Ganti, R., Ye, F., Lei, H.: Mobile crowdsensing: current state and future challenges. IEEE Commun. Mag. 49(11), 33 (2011)

9. Goldberg, R., Piccirillo, M., Nicklaus, J., Skillington, A., Lenze, E., Rodebaugh, T., Kallogjeri, D., Piccirillo, J.: Evaluation of ecological momentary assessment for tinnitus severity. JAMA Otolaryngol. Head Neck Surg. 143, 700-706 (2017)

10. Grimm, K., An, Y., McArdle, J., Zonderman, A., Resnick, S.: Recent changes leading to subsequent changes: extensions of multivariate latent difference score models. Struct. Equ. Model. Multidiscip. J. 19(2), 268-292 (2012)

11. Henry, J., Galvez, G., Turbin, M., Thielman, E., McMillan, G., Istvan, J.: Pilot study to evaluate ecological momentary assessment of tinnitus. Ear Hear. 32(2), 179 (2012)

12. Hiller, W., Goebel, G.: Rapid assessment of tinnitus-related psychological distress using the Mini-TQ. Int. J. Audiol. 43(10), 600-604 (2004)

13. Husen, K., Rafaeli, E., Rubel, J., Bar-Kalifa, E., Lutz, W.: Daily affect dynamics predict early response in CBT: feasibility and predictive validity of EMA for outpatient psychotherapy. J. Affect. Disord. 206, 305-314 (2016)

14. Jamison, R., Raymond, S., Levine, J., Slawsby, E., Nedeljkovic, S., Katz, N.: Electronic diaries for monitoring chronic pain: 1-year validation study. Pain 91(3), 277-285 (2001)

15. Killingsworth, M., Gilbert, D.: A wandering mind is an unhappy mind. Science 330(6006), 932-932 (2010)

16. Kramer, I., Simons, C., Hartmann, J., Menne-Lothmann, C., Viechtbauer, W., Peeters, F., Schruers, K., Bemmel, A., Myin- 
Germeys, I., Delespaul, P., et al.: A therapeutic application of the experience sampling method in the treatment of depression: a randomized controlled trial. World Psychiatry 13(1), 68-77 (2014)

17. Lane, S., Heddle, N., Arnold, E., Walker, I.: A review of randomized controlled trials comparing the effectiveness of hand held computers with paper methods for data collection. BMC Med. Inform. Decis. Mak. 6(1), 23 (2006)

18. Langguth, B., Goodey, R., Azevedo, A., Bjorne, A., Cacace, A., Crocetti, A., Del Bo, L., De Ridder, D., Diges, I., Elbert, T., et al.: Consensus for tinnitus patient assessment and treatment outcome measurement: Tinnitus Research Initiative meeting, Regensburg, July 2006. Prog. Brain Res. 166, 525-536 (2007)

19. Li, H., Li, T., Wang, Y.: Dynamic participant recruitment of mobile crowd sensing for heterogeneous sensing tasks. In: 12th International Conference on Mobile Ad Hoc and Sensor Systems, pp. 136-144. IEEE (2015)

20. Luo, T., Kanhere, S., Huang, J., Das, S., Wu, F.: Sustainable Incentives for mobile crowdsensing: auctions, lotteries, and trust and reputation systems. IEEE Commun. Mag. 55(3), 68-74 (2017)

21. Ma, H., Zhao, D., Yuan, P.: Opportunities in mobile crowd sensing. IEEE Commun. Mag. 52(8), 29-35 (2014)

22. Marcano-Belisario, J., Huckvale, K., Saje, A., Porcnik, A., Morrison, C., Car, J.: Comparison of Self-Administered Survey Questionnaire Responses Collected Using Mobile Apps Versus Other Methods. The Cochrane Library, London (2015)

23. Marks, M., Hemsley, D.: Retrospective versus prospective selfrating of anxiety symptoms and cognitions. J. Anxiety Disord. 13(5), 463-472 (1999)

24. Mazurek, B., Haupt, H., Olze, H., Szczepek, A.: Stress and tinnitus from bedside to bench and back. Front. Syst. Neurosci. 6, 47 (2012)

25. McArdle, J.J., Hamagami, F.: Latent difference score structural models for linear dynamic analyses with incomplete longitudinal data. In: Collins, L.M., Sayer, A.G. (eds.) Decade of behavior. New methods for the analysis of change, pp. 139-175. American Psychological Association, Washington, DC (2001). http://dx.doi. org/10.1037/10409-005

26. McCormack, A., Edmondson-Jones, M., Somerset, S., Hall, D.: A systematic review of the reporting of tinnitus prevalence and severity. Hear. Res. 337, 70-79 (2016)

27. McNeill, C.: Tinnitus management for patients with meniere's syndrome-a novel protocol using latest technology to address hearing fluctuation. J. Hear. Sci. 7(2), 139 (2017)

28. Myin-Germeys, I., Oorschot, M., Collip, D., Lataster, J., Delespaul, P., van Os, J.: Experience sampling research in psychopathology: opening the black box of daily life. Psychol. Med. 39(9), 1533 (2009)

29. Palermo, T., Valenzuela, D., Stork, P.: A randomized trial of electronic versus paper pain diaries in children: impact on compliance, accuracy, and acceptability. Pain 107(3), 213-219 (2004)

30. Pavlović, I., Kern, T., Miklavčič, D.: Comparison of paper-based and electronic data collection process in clinical trials: costs simulation study. Contemp. Clin. Trials 30(4), 300-316 (2009)

31. Probst, T., Pryss, R., Langguth, B., Rauschecker, J., Schobel, J., Reichert, M., Spiliopoulou, M., Schlee, W., Zimmermann, J.: Does tinnitus depend on time-of-day? an ecological momentary assessment study with the TrackYourTinnitus application. Front. Aging Neurosci. 9, 253 (2017)

32. Probst, T., Pryss, R., Langguth, B., Schlee, W.: Emotion dynamics and tinnitus: Daily life data from the "TrackYourTinnitus" application. Sci. Rep. 6, 31166 (2016)

33. Probst, T., Pryss, R., Langguth, B., Schlee, W.: Emotional states as mediators between tinnitus loudness and tinnitus distress in daily life: results from the "TrackYourTinnitus" application. Sci. Rep. 6, 20382 (2016)

34. Probst, T., Pryss, R., Langguth, B., Spiliopoulou, M., Landgrebe, M., Vesala, M., Harrison, S., Schobel, J., Reichert, M., Stach, M., et al.: Outpatient tinnitus clinic, self-help web platform, or mobile application to recruit tinnitus study samples? Front. Aging Neurosci. 9, 113 (2017)

35. Pryss, R., Probst, T., Schlee, W., Schobel, J., Langguth, B., Neff, P., Spiliopoulou, M., Reichert, M.: Mobile crowdsensing for the juxtaposition of realtime assessments and retrospective reporting for neuropsychiatric symptoms. In: 30th IEEE Int'1 Symposium on Computer-Based Medical Systems. IEEE Computer Society Press (2017)

36. Pryss, R., Reichert, M., Herrmann, J., Langguth, B., Schlee, W.: Mobile crowd sensing in clinical and psychological trials-a case study. In: 28th IEEE Int'l Symposium on Computer-Based Medical Systems. IEEE Computer Society Press (2015)

37. Pryss, R., Reichert, M., Langguth, B., Schlee, W.: Mobile Crowd Sensing Services for Tinnitus Assessment, Therapy and Research. In: IEEE 4th Int'1 Conf on Mobile Services. IEEE Computer Society Press (2015)

38. Pryss, R., Schlee, W., Langguth, B., Reichert, M.: Mobile crowdsensing services for tinnitus assessment and patient feedback. In: IEEE 6th Int'l Conference on Mobile Services. IEEE Computer Society Press (2017)

39. Ruf-Leuschner, M., Brunnemann, N., Schauer, M., Pryss, R., Barnewitz, E., Liebrecht, M., Kratzer, W., Reichert, M., Elbert, T.: The KINDEX-App-an instrument for assessment and immediate evaluation of psychosocial risk factors in pregnant women in daily practice by gynecologists. Midwives Gynecol. Hosp. Verhalt. 26(3), 171-181 (2016)

40. Santangelo, P., Bohus, M., Ebner-Priemer, U.: Ecological momentary assessment in borderline personality disorder: a review of recent findings and methodological challenges. J. Pers. Disord. 28(4), 555-576 (2014)

41. Schickler, M., Pryss, R., Reichert, M., Schobel, J., Langguth, B., Schlee, W.: Using mobile serious games in the context of chronic disorders-a mobile game concept for the treatment of tinnitus. In: 29th IEEE Int'1 Symposium on Computer-Based Medical Systems, pp. 343-348 (2016)

42. Schlee, W., Pryss, R., Probst, T., Schobel, J., Bachmeier, A., Reichert, M., Langguth, B.: Measuring the moment-to-moment variability of tinnitus: the TrackyourTinnitus smart phone app. Front. Aging Neurosci. 8, 294 (2016)

43. Schneck, A., Kalle, S., Pryss, R., Schlee, W., Probst, T., Langguth, B., Landgrebe, M., Reichert, M., Spiliopoulou, M.: Studying the potential of multi-target classification to characterize combinations of classes with skewed distribution. In: 30th IEEE Int'1 Symposium on Computer-Based Medical Systems. IEEE Computer Society Press (2017)

44. Shiffman, S.: Ecological momentary assessment (EMA) in studies of substance use. Psychol. Assess. 21(4), 486 (2009)

45. Shu, L., Chen, Y., Huo, Z., Bergmann, N., Wang, L.: When mobile crowd sensing meets traditional industry. IEEE Access 5, 15300 15307 (2017)

46. Trull, T., Ebner-Priemer, U.: Ambulatory assessment. Annu. Rev. Clin. Psychol. 9, 151-176 (2013)

47. Wenze, S., Miller, I.: Use of ecological momentary assessment in mood disorders research. Clin. Psychol. Rev. 30(6), 794-804 (2010)

48. Wilson, M., Kallogjeri, D., Joplin, C., Gorman, M., Krings, J., Lenze, E., Nicklaus, J., Spitznagel Jr., E., Piccirillo, J.: Ecological momentary assessment of tinnitus using smartphone technology a pilot study. Otolaryngol Head Neck Surg. 152, 897-903 (2015). (p. 0194599815569692) 